\title{
II paziente con rene policistico negli ambulatori italiani
}

Giornale di Tecniche Nefrologiche e Dialitiche 2019, Vol. 31(2) II8-123

(C) The Author(s) 2019

Article reuse guidelines:

sagepub.com/journals-permissions DOI: $10.1177 / 0394936219847894$

journals.sagepub.com/home/gtn

\section{@SAGE}

\section{Emanuela Dorigo e Luisa Sternfeld Pavia}

\section{Abstract \\ ADPKD patients in the Italian clinics}

Autosomal dominant polycystic kidney disease (ADPKD) is the most common genetic kidney disease. PKD is a multisystem disorder associated with multiple bilateral renal cysts, slowly increasing kidney size and progressive chronic kidney disease. Approximately $50 \%$ of individuals with PKD will require renal replacement therapy by the sixth decade of life.

More than $80 \%$ will also have multiple liver cysts, which can lead to local pressure effects. Cerebral haemorrhage, secondary to rupture of a berry aneurysm, occurs in up to $8 \%$ of individuals. Mitral valve prolapse occurs in up to $25 \%$ of patients.

Patients with ADPKD have a worsening in the quality of life and a burden of disease similar to cancer patients.

The quality of correct information given to patients and a warm reception have a positive impact on patients with ADPKD and contribute to an efficacious treatment program.

AIRP conducted a survey to investigate the ADPKD patient journey, meaning the personal experience and expectations of people regarding factors such as disease management, relationships with the nephrologists and their team, and therapies.

The survey was conducted on 370 people with ADPKD, using computer-assisted web interviewing (CAWI).

The results show that patients with ADPKD with a good relationship with their care team, both nephrologist and nurse, respond better to therapies.

Warm reception, dialogue, communication, support, clear explanation, reassurance and guidance altogether contribute to a successful treatment of every single person with ADPKD.

\section{Keywords}

ADPKD, Quality of life, Warm reception, Right information, Care team, Patient-doctor relationship

\section{Introduzione}

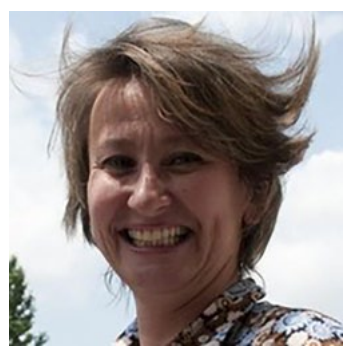

Il rene policistico autosomico dominante (autosomal dominant polycystic kidney disease, ADPKD) è una malattia multisistemica e progressiva caratterizzata da formazione di cisti ed ingrossamento dei reni e di altri organi (fegato, pancreas, milza). Fino al 50\% dei pazienti richiede terapia sostitutiva renale.

La qualità dell'accoglienza e dell'informazione contribuiscono ad un efficace percorso di assistenza al paziente con il rene policistico.

AIRP ha realizzato un'indagine presso i propri iscritti per verificare il Patient Journey, l'impatto della patologia nell'esperienza del vissuto quotidiano dei pazienti presso gli ambulatori italiani.

\section{Metodologia}

L'indagine è stata realizzata da AIRP tramite metodologia CAWI (computer-assisted web interviewing) su 370 pazienti residenti in Italia con ADPKD. Il sondaggio è stato online dal 3/12/2018 al 7/01/2019 ed i canali di

'Senior Project Manager T\&C srl, Milano, Italy

${ }^{2}$ Associazione Italiana Rene Policistico Onlus, Italy

\section{Corrispondenza:}

Emanuela Dorigo, Senior Project Manager T\&C srl, Via Ciovasso 4, 20I2I Milano, Italy.

E-mail: emanuela.dorigo@tccompany.it 


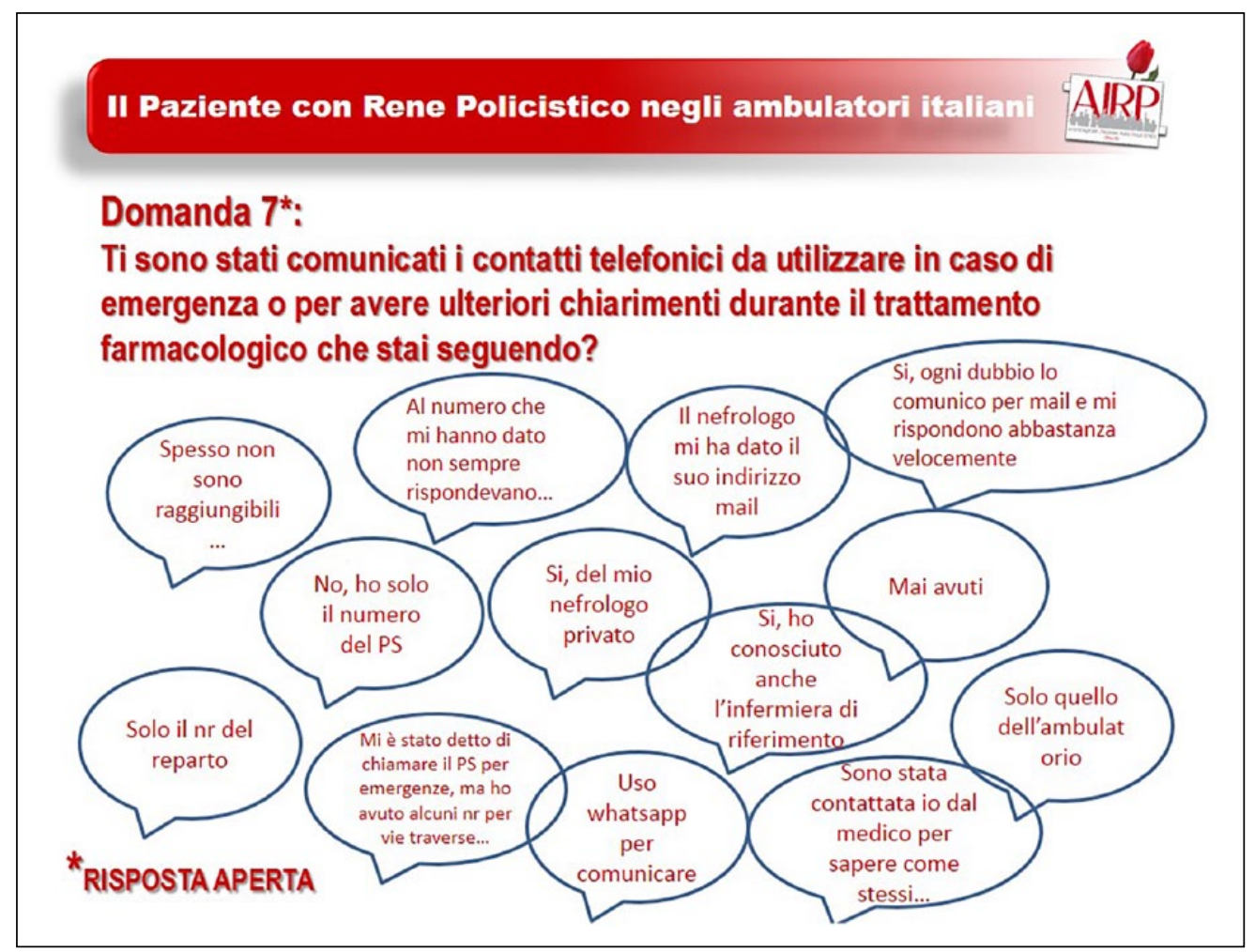

Figura I. Comunicazione riguardo i recapiti telefonici degli ambulatori.

somministrazione sono stati la Newsletter e la pagina Facebook di AIRP.

\section{Principali risultati}

Nel sondaggio, su 370 persone il $60 \%$ erano femmine, con il $47 \%$ di provenienza dal Nord Italia.

Per quanto riguarda gli ambienti in cui viene effettuata la visita, circa il $68 \%$ si è dichiarato soddisfatto, così come per circa il $74.5 \%$ il tempo di attesa della visita è stato idoneo. Per il $77 \%$ l'orario dell'appuntamento è stato rispettato.

Per quanto riguarda la tipologia di informazioni ricevute, l'aspetto è stato indagato su più piani:

1. Ritieni di aver ricevuto informazioni esaustive e chiare rispetto alla malattia e alla cura?

2. Ti sei sentito libero di esprimere dubbi, perplessità e di porre delle domande?

3. Hai avuto la sensazione che il nefrologo abbia dedicato solo a te il tempo del colloquio/visita? (ha silenziato il cellulare, ti guardava negli occhi, era interessato a ciò che dicevi...)

A tutte e tre le domande la maggioranza, rispettivamente $81.4 \%, 87.3 \%$ e $85.1 \%$, ha dato un riscontro positivo.

Risposte più discordanti sono state date per quanto riguarda i contatti telefonici forniti in caso di emergenza o per avere chiarimenti durante il trattamento farmacologico, a sottolineare la disparità sul territorio italiano (figura 1).
È stato indagato anche il rapporto tra i medici nefrologi dei centri ed il Medico di Medicina Generale. Dalle risposte degli intervistati, non sembra esserci grande comunicazione tra ospedale e territorio, perché il 53.2\% dichiara di non avere ricevuto l'informativa per il Medico di Medicina Generale.

Questo dato sottolinea come ancora molto ci sia da fare per costruire una collaborazione tra gli specialisti ed il Medico di Base, che conosce la storia del singolo paziente e le sue problematiche al di là della questione nefrologica. I medici di base (tranne qualche caso isolato) hanno scarsa conoscenza della patologia e sono poco di aiuto per il paziente nel supportarlo in caso di problemi. Nelle risposte aperte, ci sono un paio di casi esemplificativi:

- "i medici di base non sono informati sulla malattia, non controllano la pressione, non conoscono gli ultimi sviluppi della ricerca";

- "il medico di base è stato un prezioso supporto, molto competente riguardo la patologia ADPKD e dialisi. Purtroppo, da pochissimo ho cambiato medico di base perché il mio è andato in pensione e la sostituta sembra che non abbia idea di nulla riguardo dialisi e trapianto".

Si potrebbe pensare ad eventi educazionali di incontro/ confronto per Medici di Medicina Generale e specialisti per costruire una rete collaborativa forte a sostegno del percorso di cura del singolo paziente. Occorre sensibilizzare i Medici 


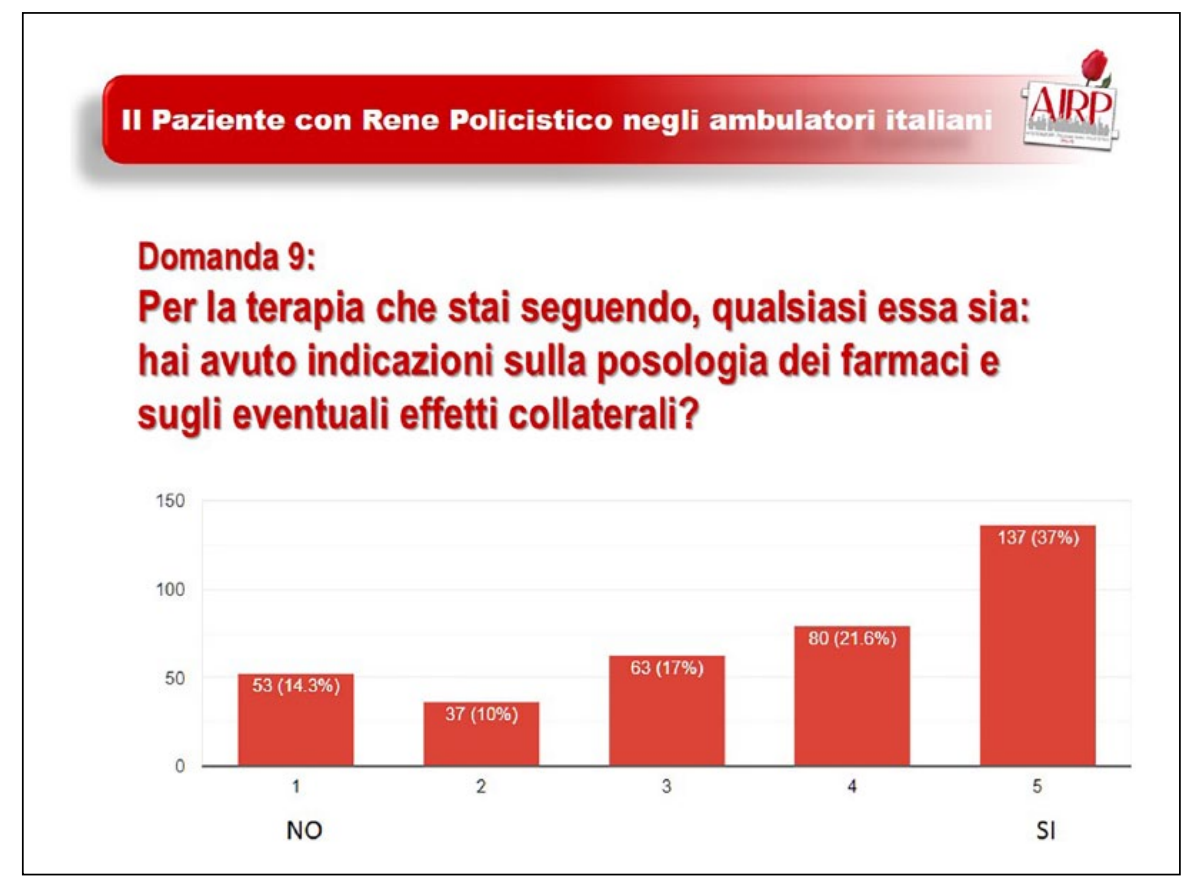

Figura 2. Comunicazione sulla terapia.

di Medicina Generale, in quanto prima figura in grado di individuare la patologia e di fare un'indagine famigliare, in modo tale da indirizzare allo specialista quanto prima.

Per quanto riguarda la terapia e le modalità di assunzione è emerso qualche problema di comunicazione maggiore (figura 2).

Per quanto riguarda i consigli pratici, come ricezione corretta delle informazioni su: adeguato apporto idrico, consigli pratici per la modalità di assunzione e informazioni in merito a stili di vita/programmi nutrizionali particolari da seguire, i risultati tracciano una situazione abbastanza positiva, anche se si può evincere che probabilmente nei centri dove è presente la figura dell'infermiere c'è un'attenzione più mirata al paziente che viene seguito più nel dettaglio. Un

infermiere dedicato che supporti anche a distanza rispondendo alle domande dei pazienti su patologia o trattamento è utile per dissipare dubbi e domande che emergono costantemente su quello che si può fare/non fare, assumere/non assumere....

Nelle testimonianze aperte risulta invece un po' di confusione riguardo la dieta e lo stile di vita:

- "Sono affetta dalla malattia, ma attualmente non ho sintomi di rilievo: seguo una dieta che favorisce un buon funzionamento ai reni";

- "Nel mio caso navigo a vista tenendo a distanza sale, cioccolata e caffè";

- "Io sto seguendo una dieta alimentare che va oltre quello che dicono i nefrologi e vedo che sto meglio".

L'argomento dialisi viene affrontato in modo difforme, o comunque la percezione degli intervistati è che non venga affrontato in modo esauriente e comprensibile (figura 3-4):

- "Ritengo che non c'è sufficiente informazione, tutto è molto superfluo";

- "In dialisi nessun supporto psicologico";

- "Non ho approfondito il discorso di dialisi, perché nonostante la patologia i miei reni per ora funzionano".

Ci sono anche degli esempi positivi:

- "Sono stata seguita bene sia in pre-dialisi (peritoneale) e durante".

- "Tutto il personale medico ed infermieristico mi ha seguito costantemente e mi segue attualmente in modo molto professionale".

L'argomento "trapianto" presenta ancora più problematiche a livello di comunicazione, come ben si evince dalle figure 5-6.

La poca chiarezza di comunicazione sulla possibilità di trapianto si affianca all'abilità personale di reagire e di elaborare/accettare la malattia, con il forte senso di gratitudine per aver ricevuto "un dono": "ho ricevuto da 8 mesi un trapianto di rene e dentro di me è tornato a risplendere il sole... sono tornata a vivere".

\section{Conclusioni}

Dall'indagine effettuata emerge un panorama variegato dei nostri ambulatori italiani (figura 7). 


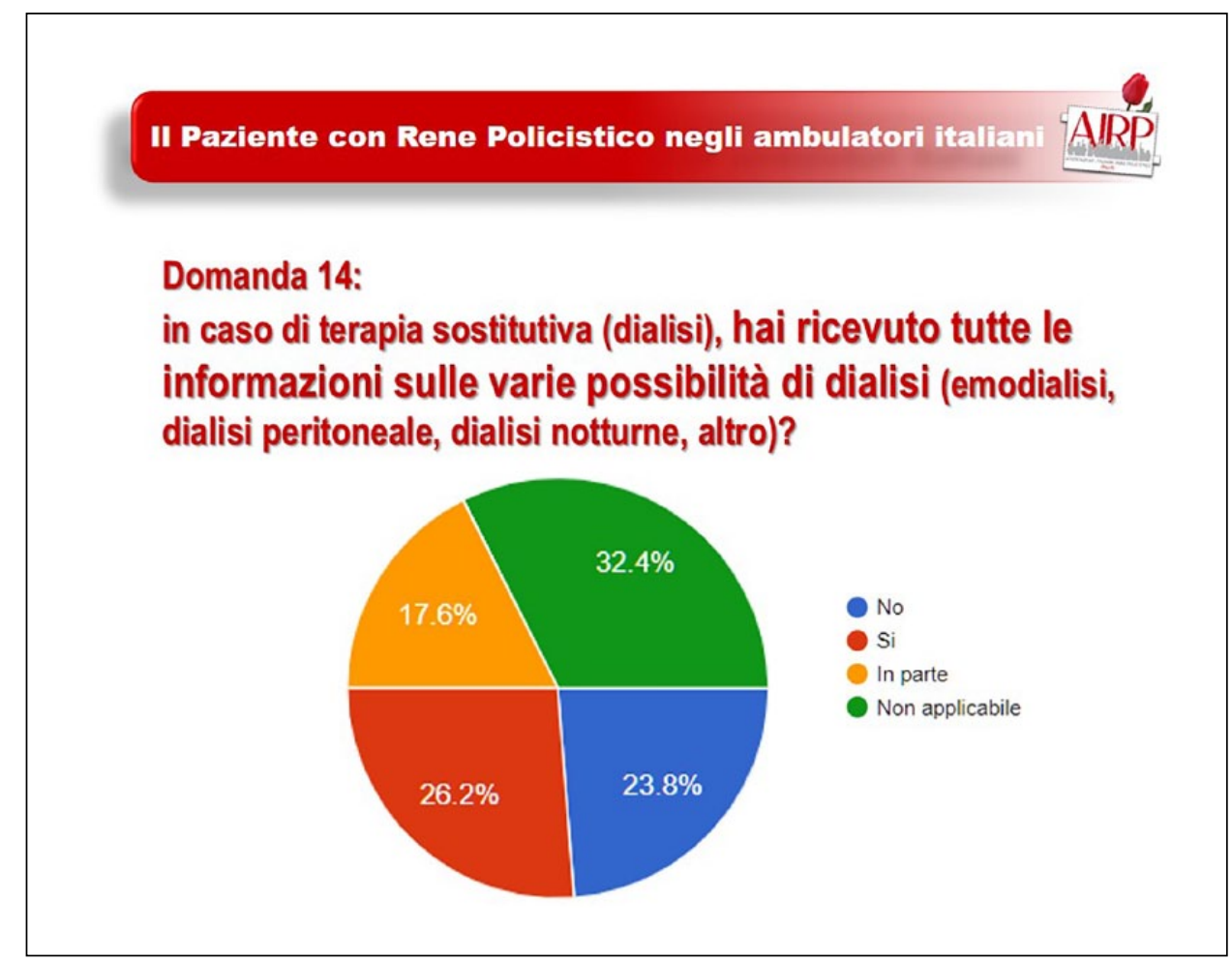

Figura 3. Il percepito sulla comunicazione in merito alla terapia sostitutiva.

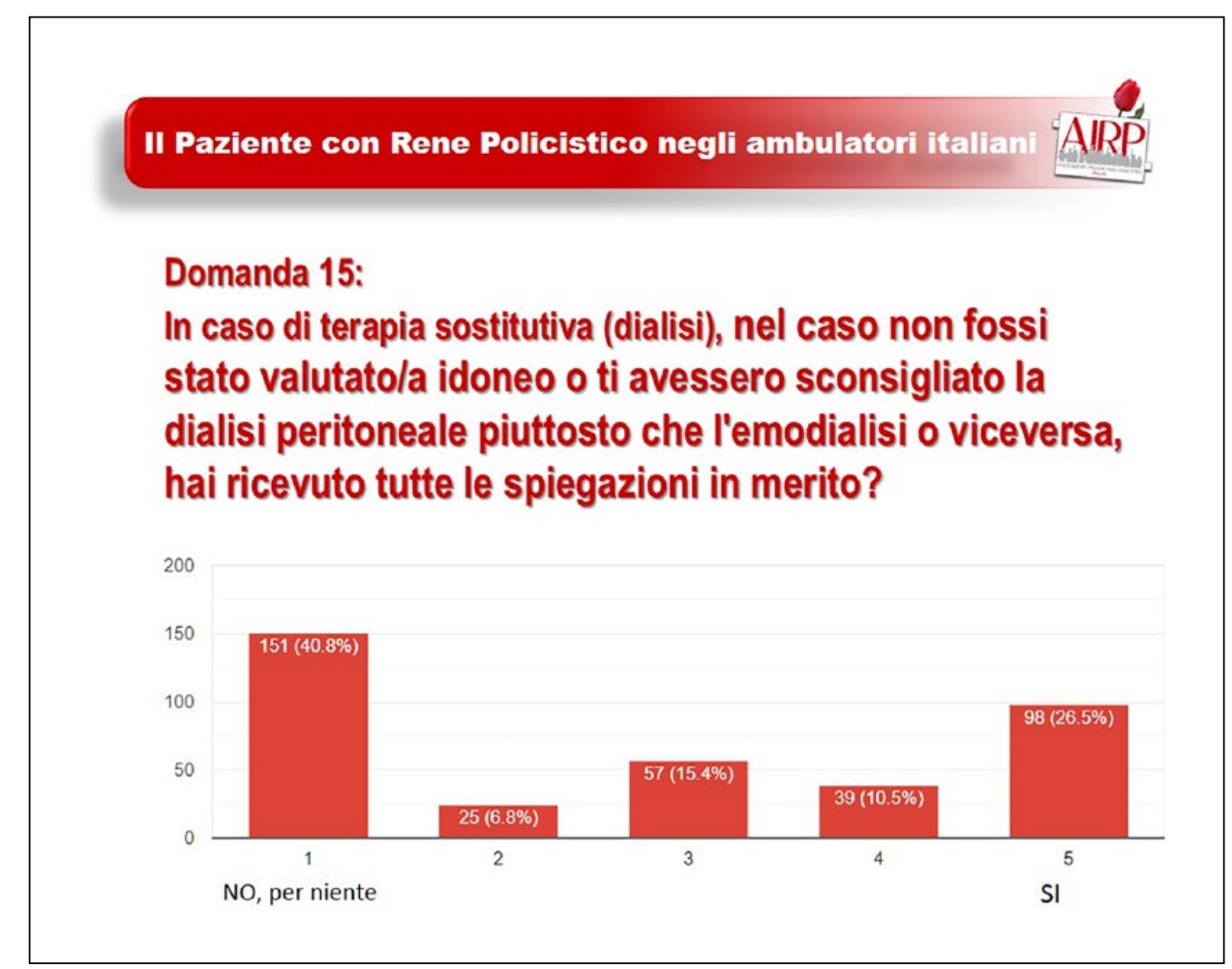

Figura 4. Il percepito sulla comunicazione in merito alla dialisi. 


\section{Paziente con Rene Policistico negli ambulatori italiani AIRP}

\section{Domanda 16:}

in caso di terapia sostitutiva (trapianto), ti hanno informato sui vari tipi di trapianto (da vivente, da cadavere, cross-over)?

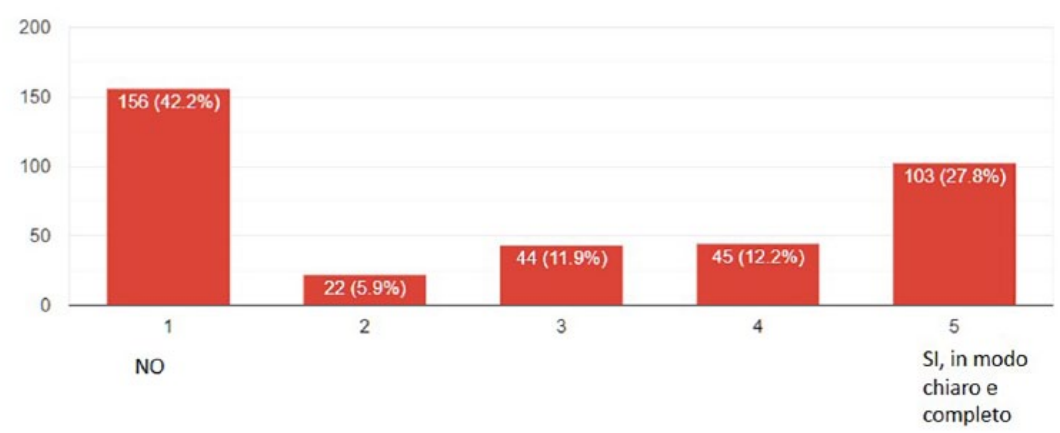

Figura 5. II percepito sulla comunicazione in merito ai vari tipi di trapianto.

II Paziente con Rene Policistico negli ambulatori italiani AIRP

Domanda 17*:

In caso di terapia sostitutiva (trapianto), sei consapevole che dovrai seguire sempre e costantemente una terapia per preservare il rene trapiantato?

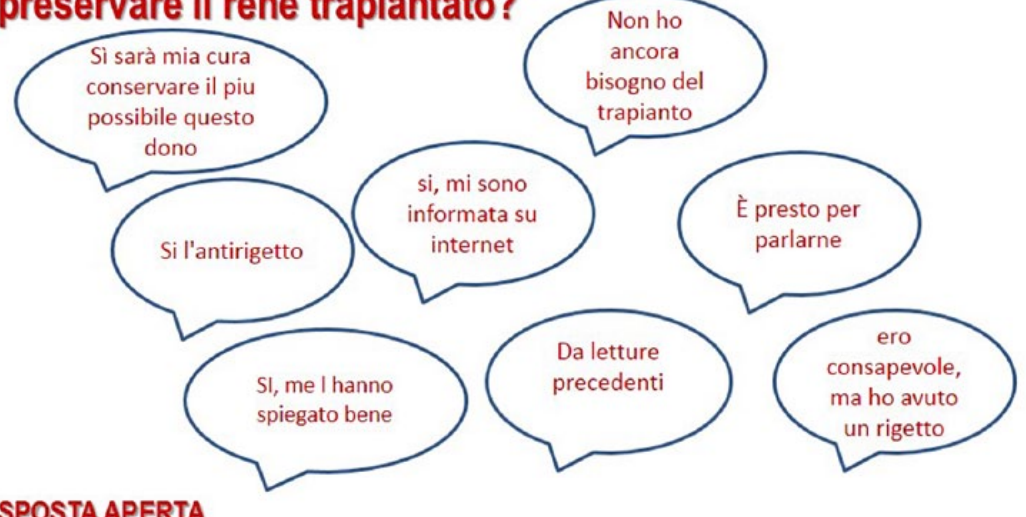

* RISPOSTA APERTA

Figura 6. Il percepito sulla comunicazione in merito alla terapia per preservare il rene trapiantato. 


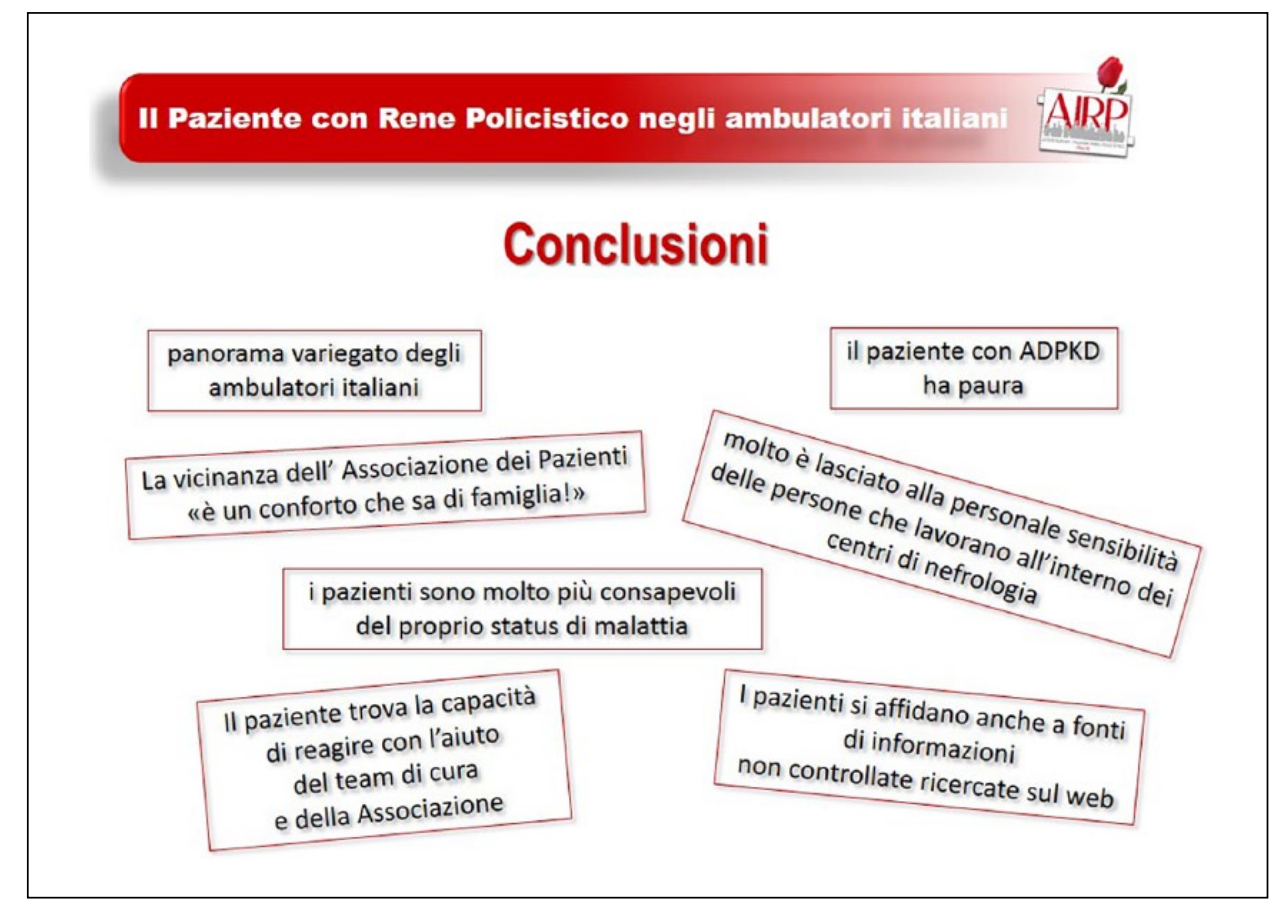

Figura 7. Resoconto in merito al sondaggio effettuato negli ambulatori italiani.

Sembrerebbe che ancora molto sia lasciato alla personale sensibilità delle persone che lavorano all'interno dei centri di nefrologia e che i pazienti molto più consapevoli del proprio status di malattia, si affidano anche a fonti di informazioni non controllate ricercate sul web. Il paziente con ADPKD ha paura, ma nella maggior parte dei casi trova la capacità di reagire con l'aiuto del team di cura e dell'Associazione Pazienti, "la cui vicinanza è un conforto che sa di famiglia!"

\section{Dichiarazione di assenza di conflitto di interessi}

L'Autore dichiara di non aver conflitti di interessi.

\section{Finanziamenti}

L'Autore dichiara di non aver ricevuto finanziamenti specifici da qualsiasi ente nel settore pubblico, privato o senza fini di lucro.

\section{Bibliografia}

1. AIRP - Associazione Italiana Rene Policistico Onlus. "Rene policistico autosomico dominante: parlarne è già combatterlo". Edizione, 2013.

2.Scolari F. "Manifestazioni renali ed extrarenali del rene policistico autosomico dominante". Edizione AIRP Associazione Italiana Rene Policistico Onlus, 2013. 